\title{
Utilizing the Crosstalk Among Minocycline, Chicoric Acid, 13-Cis Retinoic Acid(Aerosolized) and Vitamin D as a Potent Quadrate Therapy for treating coinfection with Multidrug-resistant TB and COVID-19 . A double-edged sword Clinical Study
}

Mahmoud Ramadan Elkazzaz ( $\square$ mahmoudelkazzaz2051@gmail.com )

Department of chemistry and biochemistry, Faculty of Science, Damietta University, Egypt https://orcid.org/0000-0003-3703-520X

Amr Ahmed

Director of tuberculosis program Ghubera, public health department ,First health cluster ,Ministry of health , Riyadh, Saudia Arabia

https://orcid.org/0000-0003-3477-236X

\section{Method Article}

Keywords: Multidrug-resistant TB, COVID-19, Chicoric Acid, 13-Cis Retinoic Acid, Minocycline, vitamin D, Clinical Trial Kafrelshiekh University

Posted Date: October 12th, 2021

DOl: https://doi.org/10.21203/rs.3.rs-963736/v1

License: @ (1) This work is licensed under a Creative Commons Attribution 4.0 International License. Read Full License 


\section{Abstract}

Tuberculosis (TB) is a major infectious disease killer globally. It affected 10 million and killed 1.4 million people in 2019 alone. TB is considered a disease caused by a bacterium-Mycobacterium tuberculosis-that usually attacks the lungs, but can attack any part of the body. But TB has a worrisome connection to the novel coronavirus.. Both diseases are airborne and spread when people cough or sneeze. The predicted impact of the COVID-19 pandemic is an additional 190,000 TB deaths in 2020, and it is expected in the next $5 \mathrm{y}$ that there will be up to a $20 \%$ increase in the global TB disease burden, stressing the critical need for new safe and effective drugs against Mycobacterium tuberculosis (Mtb). In addition, controlling multidrug-resistant TB (MDR-TB) presents a huge public health challenge. Recently it was showed that hospitalized patients with Tuberculosis are more susceptible to COVID-19 infection and complication. Furthermore, hospitalized patients with MDR-TB are increasingly vulnerable to COVID-19 complications than patients with non-resistant tuberculosis.. For someone with latent TB, contracting COVID-19 could activate the bacterium, potentially leading to an accelerated and more severe form of the disease WHO estimates that these COVID-19 related disruptions in access to TB care could cause an additional half a million TB deaths. Older age, especially $>65$ years, may be a risk factor for death from COVID-TB, consistent with previous findings indicating that the mortality rate from COVID-19 increases exponentially with age. Thus, the elderly should be the primary focus of both COVID-19 and COVID-TB mitigation efforts due to its much higher mortality risk in that group. COVID-TB patients had a much higher rate of comorbidities than COVID-19 patients At present, evidence suggests that the main transmission route of both COVID-19 and TB is via respiratory droplets, and their main target are the lungs, which can lead to a worse outcome among COVID-19 and TB coinfection patients (aptly abbreviated COVID-TB). As a result, coinfections with common viral and bacterial (COVID-TB) pathogens among hospitalized patients are a severe concern that will likely worsen patient outcomes and pose a real challenge for treating those patients.

\section{Conclusions}

New drug discovery could require several years with no guarantee but repurposing established drugs may be useful to treat confection with COVID-19 and Nonresistant Strains of Mycobacterium tuberculosis: or resistant Strains of Mycobacterium tuberculosis. Here we demonstrate that we could utilize the crosstalk among Chicoric Acid, 13-Cis Retinoic Acid, Minocycline and vitamin D as a novel quadrate therapy against Multidrug-resistant TB and COVID-19 coinfection.

\section{Introduction}

Tuberculosis (TB) is a leading cause of morbidity and ranks among the 10 most common causes of death worldwide. It killed 1.5 million people in 2018, and about 10 million people fall ill with it each year, according to the World Health Organization (WHO). While it's mostly curable and preventable, some strains of the bacteria have proven to be drug resistant(1). MDR-TB is a type of tuberculosis (TB) infection produced by bacteria resistant to at least two of the most powerful first-line anti-TB treatments, isoniazid and rifampin(2). The estimated global number of multidrug-resistant (MDR) and extensively drug-resistant (XDR)-TB cases among newly diagnosed patients with pulmonary TB in the year 2012 was 450000 (range 300 000-600 000)(3). Infectious diseases caused by mycobacteria differ from almost all other bacterial infections by the requirement for a very long duration of treatment in order to achieve relapse-free cure with the currently available therapies(4). This is especially the case for TB when M. tuberculosis is MDR (i.e. resistant to the most potent anti-TB drugs rifampicin and isoniazid)(4). What makes the problem worse is that patients with MDR-TB are more prone to COVID-19. According to WHO, the COVID-19-related disruptions in TB care could result in an additional half-million TB fatalities(3,5). Contracting COVID-19 could activate the bacterium in patients who has latent tuberculosis, perhaps leading to a faster and more severe version of the disease. Therefore those patients may be more susceptible to SARS-CoV-2 infection, and that COVID-19 disease progression may be more rapid and severe. Given that TB causes more deaths than any other infectious disease (1.45 million deaths and 10 million new cases in 2018(6), and that global rates of LTBI are estimated to be as high as $25 \%(6)$, these findings are a cautionary reminder to clinicians that MTB infection status should be considered when treating. There is growing evidence to suggest that previous or current TB infection or disease are associated with poor COVID-19 outcomes $(7,8)$ including an approximately two- to three-fold increase in mortality (which occurred more quickly) and a $25 \%$ relative decrease in the possibility of recovery (which occurred more slowly) for COVID-19 coinfection with current TB disease. $(9,10)$. ). It is clear that, coinfections with both COVID-19 and tuberculosis (COVID-TB) pathogens, or (COVIDMultidrug-resistant TB) pathogens among hospitalized patients are a severe concern that will likely worsen patient outcomes and represent a real challenge for treating those patients... Here we demonstrate that we could utilize the crosstalk among Chicoric Acid, 13Cis Retinoic Acid, Minocycline, and vitamin D as a novel quadrate therapy against Multidrug-resistant TB and COVID-19 confection .

\section{I) Part of Tuberculosis:-}


Tuberculosis (TB) is a significant infectious disease that kills people all over the world. In just one year, it afflicted ten million people and killed 1.4 million. The COVID-19 pandemic is likely to result in an additional 190,000 TB fatalities in 2020, and the worldwide TB disease burden is expected to rise by up to $20 \%$ in the next five years, highlighting the crucial need for new safe and effective treatments against Mycobacterium tuberculosis (Mtb). Controlling multidrug-resistant tuberculosis (MDR-TB) is also a major public health issue. New medication development could take several years with little guarantee, however repurposing current drugs could be effective in treating tuberculosis patients. In our protocol that we highlight on the possibility of repurposing Chicoric Acid, 13-Cis Retinoic Acid, Minocycline, and vitamin $D$ as a novel quadrate therapy against Drug-resistant tuberculosis. Drug-resistant tuberculosis represents a global emergency, requiring new drugs. Recently Minocycline was found to be highly potent in laboratory strains of Mycobacterium TB, and 30 drug-sensitive and multidrug/extensively drug-resistant clinical strains were susceptible to clinically attainable dosages(12). The lung concentration-time profiles of a $7 \mathrm{mg} / \mathrm{kg} /$ day human-equivalent minocycline dosage yielded bacterial kill rates comparable to first-line antituberculosis drugs(12). Extracellular bacilli were destroyed directly by minocycline. Minocycline also killed intracellular bacilli indirectly through granzyme A-driven apoptosis(12). Furthermore, minocycline showed dose-dependent anti-inflammatory effect, suggesting that it may protect tuberculosis patients against immunopathology(12). A study showed that $\mathrm{M}$. tuberculosis induced the expression of indoleamine 2,3-dioxygenase (IDO), an enzyme involved in tryptophan catabolism, in macrophages and in the lungs of animals (mice and macaque) with active disease(13). In a macaque model of inhalation TB, suppression of IDO activity reduced bacterial burden, pathology, and clinical signs of TB disease, leading to increased host survival. This increased protection was accompanied by increased lung T cell proliferation, induction of inducible bronchus-associated lymphoid tissue and correlates of bacterial killing (13). A recent study showed that Minocycline-induced significantly inhibition of IDO expression(14). But Minocycline-induced inhibition of IDO expression is retinoiddependent(14). The combined treatment with minocycline and retinol, however, resulted in a striking, statistically significant decrease in IDO(14). Co-treatment with minocycline and retinol again resulted in decreased TNF-a and IL-6 levels(14). A study showed that IL-6 inhibits IFN- $y$ induced autophagy in Mycobacterium (TB) H37Rv infected macrophages(15). As well as neutralization of endogenous IL-6 by anti-IL-6 antibody significantly enhances the IFN- $\gamma$ mediated killing of the intracellular bacteria(15). Minocycline's anti-inflammatory effects are mediated through RAR signaling(14). Therefore, The combined treatment with minocycline and retinol is expected to effectively inhibit (TB) and its inflammatory complication, All-trans-retinoic acid (RA), an active form of vitamin A, has the ability to reduce human mortality through effects that are considered to be related to the immune system (16). Vitamin A deficiency results in multiple abnormalities of innate and adaptive immunity involving cell differentiation, hematopoiesis, and blood and lymphoid organ cell populations, and the organism's ability to respond to challenges by pathogens, antigens, and mitogens $(17,18)$. Fortunately, Retinoic Acid significantly inhibits the in vivo growth of $\mathrm{M}$. tuberculosis and the development of tuberculosis(19).In addition to, 13-Cis RA and Chicoric Acid ( CA ) enhanced the cell surface expression of HLA-DR and CD14 molecules on U937 macrophages and prevented the growth of Mtb within macrophages(20). Moreover, 13-cis RA and CA, have increased NO generation compared to untreated control macrophages, significantly(20). Both drugs have a significant inhibitory effect on Mtb growth but CA at the highest concentration was more potent than 13-cis RA(20). Therefore we will use retinoic acid to induce the effect of Minocycline as well as its ability to inhibit tuberculosis in combination with CA .Recent data showed that Vitamin D support innate immune responses to Mycobacterium TB and Low vitamin D levels were associated with a 5 -fold increased risk for progression to tuberculosis(21). Deficiency of vitamin $D$ has long been implicated in activation of (TB) (21). Serum vitamin D in TB patients are lower than in healthy controls(21). Vitamin D has been found to speed up the clearance of (TB) bacteria from the lungs of people with multi-drug resistant TB, according to a study of 1,850 patients(22). It was showed that Vitamin $\mathrm{D}$ receptor (VDR) must form a heterodimer complex with retinoid $\mathrm{X}$ receptor (RXR) to regulate gene transcription(23). While little is known of the molecular determinants of basal expression of the VDR in cells, the VDR gene is known to be regulated by a variety of hormones including PTH, retinoic acid, and the glucocorticoids (23). Retinol plays a crucial role in lung development and signaling the vitamin D pathway. 9-cis-retinoic acid, an active vitamin A metabolite and the ligand of RXR, assists VDR signaling and suppresses the degradation of circulating vitamin $D(24)$.

\section{I) Part of COVID-19}

Before the COVID-19 outbreak, TB had been the most fatal infectious disease in the world for many years (25). Globally, an estimated 10 million people contracted TB and 1.4 million died from TB in 2019 (25). At present, evidence suggests that the main transmission route of both COVID-19 and TB is via respiratory droplets, and their main target are the lungs, which can lead to a worse outcome among COVID19 and TB coinfection patients (aptly abbreviated COVID-TB) $(26,25)$. Older age, especially $>65$ years, may be a risk factor for death from COVID-TB, consistent with previous findings indicating that the mortality rate from COVID-19 increases exponentially with age $(27,28,25)$. COVID-TB patients had a much higher rate of comorbidities than COVID-19 patients (56.41 vs. $25.1 \%$ ) (29). Therefore, due to the high prevalence of both of these infectious diseases and the potential worse prognosis of coinfection, an intensive investigation of COVID-TB cases may be of great clinical significance $(30,31,25)$.Drug repurposing or repositioning is a well-known strategy that seeks to deploy existing licensed drugs for newer indications and provides the quickest possible transition from bench to clinics for unmet therapeutic 
needs. Given the current, urgent, and dire need for effective therapies against novel coronavirus-19 and confection with both COVID-19 and multidrug-resistant tuberculosis (MDR-TB), this approach is particularly appealing. Here we indicate that this quadrate therapy used as a potent treatment against multidrug-resistant tuberculosis (MDR-TB) could be used against COVID-19.

Because of the potent documented anti-inflammatory features of minocycline and its well-known safety and interaction profile, relatively low costs, and widespread availability It offers distinct advantages in terms of potential efficacy in patients with life-threatening acute respiratory distress syndrome (ARDS) and myocardial injury. Minocycline is a semi-synthetic tetracycline analogue with broad-spectrum activity against gram-positive and gram-negative bacteria, Rickettsia, Mycoplasma pneumoniae, Chlamydia, and Plasmodium spp., as well as a variety of spirochetes(32,33). The anti-inflammatory and immunomodulatory effects of minocycline may be useful in the treatment of COVID-19 patients, especially if they have respiratory issues such ARDS or multiorgan damage. As previously noted, COVID19-associated ARDS is caused by a cytokine storm, with IL- 6 and other cytokines playing a critical role in inducing organ damage and mortality. Minocycline has been shown to suppress the secretion of cytokines such as IL-6, IL-2, and TNF-, suggesting that it may be useful in the treatment of cytokine release syndrome caused by disease.. $(34,35,36)$. Furthermore, cell cultures treated with minocycline showed considerable suppression of activated phagocytes and IF- expression in CD8 + T cells(37). Chemically modified tetracyclines (CMT), particularly CMT-3, significantly delayed the development of acute respiratory distress syndrome (ARDS) and prolonged survival in treated animals compared to the control group in several preclinical studies involving acute respiratory distress syndrome models. Tetracycline treatment was also effective in decreasing ARDS histological lesions in these animals(38,39). Even when used chronically at doses up to $200 \mathrm{mg} /$ day, the highest approved dosage, the drug is generally safe and well-tolerated by patients $(40,41)$.It was showed that Minocycline's anti-inflammatory effects are mediated through RAR signaling(14). Therefore, The combined treatment with minocycline and retinol is expected to effectively inhibit COVID-19 and its inflammatory complication. Interestingly, it was that 13 cis retinoic acid protect individuals treated for acne against COVID-19. Our clinical trial on assessing the efficacy and safety of 13 cis retinoic acid against COVID-19 was the first clinical study in literature indicted the possibility of repurposing 13 cis retinoic acid against COVID-19. This clinical study is the first clinical study in literature (submitted on 20 April, 2020) which demonstrated that Isotretinoin will provide complete protection against COVID-19

(ClinicalTrials.gov Identifier: NCT04353180).

In addition to our clinical study in which we suggested that aerosolized all trans retinoic acid will potentially act against COVID-19 (ClinicalTrials.gov Identifier: NCT04396067)( https://covid19-help.org/substance/tretinoin).

Isotretinoin, a retinoid derivative of vitamin A, is used in the treatment of severe acne. It is the strongest down-regulator of ACE-2 receptors. It has been documented that isotretinoin is a protease inhibitors and can be a taken as a target therapy in COVID-19(42). After our clinical trial many studies confirmed our suggestion and found that isotretinoin and all trans retinoic acid could protect individuals against COVID-19 complication $(43,44,45,46,47,48)$.

Vitamin D, a steroid and versatile hormone, plays crucial roles in phosphorus-calcium metabolism and in the immune system of both humans and animals(50)Numerous studies have revealed a wide range of pharmacological and physiological functions of vitamin D. It has anti-inflammatory, antioxidant, and antiviral effects. Besides this, it has crucial regulatory roles in the adaptive and innate immune systems.(51)It was shown that vitamin D deficiency is quite common among COVID-19 patients(49,52).A recent clinical study showed that COVID-19 patients supplemented with vitamin D had less severe symptoms of the disease(53).Another compound, chicoric acid, has also shown potent antiviral effects against herpes simplex, influenza, enterovirus, hepatitis B virus, HIV, vaccinia virus, and vesicular stomatitis virus (VSV)-Ebola (54). In HIV-1, chicoric acid and its derivatives inhibit the enzyme integrase, the function of which is the integration of the viral DNA into the host genome, leading to the replication of virus (55). Treatment with Echinacea has been shown to retain the activity of NK cells and monocytes, which provides nonspecific immunity and eliminates virus-containing cells (56). E. purpurea roots showed antiviral activity against influenza, herpes virus, and VSV in resistant mouse L929 cells (57).

\section{Study description}

This double blinded, randomized, placebo controlled trial on the clinical efficacy and antimicrobial mechanisms of quadrate therapy (Chicoric Acid, 13-Cis Retinoic Acid(Aerosolized), Minocycline and Vitamin D) as a Potent Quadrate Therapy Against confection with COVID-19 and MDR-TB Tuberculosis. 200 men and women will be enrolled, who are age 18 to 65 years inclusive, have at least two positive sputum smears for tuberculosis (TB), and have given informed consent to participate in the study. All of the enrollees will have active MDR-TB and COVID-19. The effect of the quadrate therapy status will be examined in the context of a randomized trial conducted in Egyptian or Saudi Arabian Subjects will be randomized to receive either the quadrate therapy or placebo from the start of their TB therapy, through the 1 months of anti-TB therapy, and until the last recruited patient reaches 6 months of follow up. Thus, the first subject

Page $4 / 10$ 
is likely to be followed for 12 months, assuming the duration of recruitment is 12 months. The endpoints of interest include bacteriologic cure, immune response parameters, and clinical outcomes. The researchers will also examine the utility of these immune response parameters as surrogate markers for treatment efficacy in TB, irrespective of the quadrate therapy and other risk factors. The study will be carried out as a collaborative effort between. Kafr elshiekh university ,Faculty of Medicine, Egypt and First health cluster ,Ministry of health ,Saudia Arabia. Subjects will be randomly assigned to receive A) Minocycline i.v. 4,5 mg/kg gradual in 2 divided doses increases from $4,5 \mathrm{mg} / \mathrm{kg}$ from the first week to 7.5 and $10 \mathrm{mg} / \mathrm{kg}$ to the last week. In addition to Aerosolized 13 cis retinoic acid or All trans retinoic acid or 9 cis retinoic acid gradual in 2 divided doses increases from $0.2 \mathrm{mg} / \mathrm{kg} /$ day to $4 \mathrm{mg} / \mathrm{kg} /$ day as inhaled retinoic acid therapy for 30 days. Moreover, the patients will receive Echinacea Purpurea Extract Capsules Polyphenols Chicoric Acid .Chicoric acid (also known as cichoric acid and dicaffeoyltar- taric acid) is the main phenolic compound found in Echinacea purpurea. Echinacea phytochemical profile Each tablet is comprised of the equivalent of $1275 \mathrm{mg}$ of echinacea root, as follows: A) Echinacea purpurea - $675 \mathrm{mg}$ root yields $112.5 \mathrm{mg}$ dried extract, standardized to contain $2.1 \mathrm{mg}$ alkamides. Each batch of tablets Caffeic acid derivative levels are typically as follows: caftaric acid $=0.5$ to $2.0 \mathrm{mg} /$ tablet; cichoric acid $=3.4$ to $8.5 \mathrm{mg} /$ tablet; chlorogenic acid $=<0.5 \mathrm{mg} /$ tablet; dicaffeoyl quinic acids (including cynarin) $=0.7$ to $2.0 \mathrm{mg} /$ tablet; echinacoside $=1.0$ to $1.7 \mathrm{mg} /$ tablet. Furthermore, the patients will receive

\section{Material And Methods}

\section{Active Comparator: 13 cis retinoic acid, Minocycline, Chicroic Acid and Vitamin D for (MDR-TB)}

50 subjects will be randomly assigned to receive A) Minocycline i.v. 4,5 mg/kg gradual in 2 divided doses increases from 4,5 mg/kg from the first week to 7.5 and $10 \mathrm{mg} / \mathrm{kg}$ to the last week. In addition to Aerosolized 13 cis retinoic acid in gradual 2 divided doses increases from $0.2 \mathrm{mg} / \mathrm{kg} /$ day to $4 \mathrm{mg} / \mathrm{kg} /$ day as inhaled retinoic acid therapy for 30 days. Moreover, the patients will receive Echinacea Purpurea Extract Capsules Polyphenols Chicoric Acid. Echinacea phytochemical profile Each tablet is comprised of the equivalent of $1275 \mathrm{mg}$ of echinacea root, as follows: A) Echinacea purpurea - $675 \mathrm{mg}$ root yields $112.5 \mathrm{mg}$ dried extract, standardized to contain $2.1 \mathrm{mg}$ alkamides. Each batch of tablets contain choric acid choric acid $=3.4$ to $8.5 \mathrm{mg} /$ tablet.. Furthermore, the patients will receive Cholecalciferol(Vitamin D) Intramuscular injection of 600,000 units of Cholecalciferol for 2 doses given at week 0 and week 4

\section{Active Comparator: 9 cis retinoic acid, Minocycline,Chicroic Acid and Vitamin D for (MDR-TB)}

50 subjects will be randomly assigned to receive A) Minocycline i.v. 4,5 mg/kg gradual in 2 divided doses increases from 4,5 mg/kg from the first week to 7.5 and $10 \mathrm{mg} / \mathrm{kg}$ to the last week. In addition to Aerosolized 9 cis retinoic acid in gradual 2 divided doses increases from $0.2 \mathrm{mg} / \mathrm{kg} /$ day to $4 \mathrm{mg} / \mathrm{kg} /$ day as inhaled retinoic acid therapy for 30 days. Moreover, the patients will receive Echinacea Purpurea Extract Capsules Polyphenols Chicoric Acid. Echinacea phytochemical profile Each tablet is comprised of the equivalent of $1275 \mathrm{mg}$ of echinacea root, as follows: A) Echinacea purpurea - $675 \mathrm{mg}$ root yields $112.5 \mathrm{mg}$ dried extract, standardized to contain $2.1 \mathrm{mg}$ alkamides. Each batch of tablets contain choric acid $=3.4$ to $8.5 \mathrm{mg} /$ tablet. Furthermore, the patients will receive Cholecalciferol(Vitamin D) Intramuscular injection of 600,000 units of Cholecalciferol for 2doses given at week 0 and week 4

\section{Active Comparator: All trans retinoic acid, Minocycline,Chicroic Acid and Vitamin D for (MDR-TB)}

50 subjects will be randomly assigned to receive A) Minocycline i.v. 4,5 mg/ $\mathrm{kg}$ gradual in 2 divided doses increases from 4,5 mg/ $\mathrm{kg}$ from the first week to 7.5 and $10 \mathrm{mg} / \mathrm{kg}$ to the last week. In addition to Aerosolized All trans retinoic acid in gradual 2 divided doses increases from $0.2 \mathrm{mg} / \mathrm{kg} /$ day to $4 \mathrm{mg} / \mathrm{kg} /$ day as inhaled retinoic acid therapy for 30 days. Moreover, the patients will receive Echinacea Purpurea Extract Capsules Polyphenols Chicoric Acid. Echinacea phytochemical profile Each tablet is comprised of the equivalent of $1275 \mathrm{mg}$ of echinacea root, as follows:A) Echinacea purpurea - $675 \mathrm{mg}$ root yields $112.5 \mathrm{mg}$ dried extract, standardized to contain $2.1 \mathrm{mg}$ alkamides. Each batch of tablets contain choric acid $=3.4$ to $8.5 \mathrm{mg} /$ tablet. Furthermore, the patients will receive Cholecalciferol(Vitamin D) Intramuscular injection of 600,000 units of Cholecalciferol for 2 doses given at week 0 and week 4

Active Comparator: 13 cis retinoic acid, Minocycline, Chicroic Acid and Vitamin D For (COVID-19 and MDR-TB)

50 subjects with confection of COVID-19 and multidrug-resistant tuberculosis (MDR-TB) will be randomly assigned to receive A) Minocycline i.v. 4,5 mg/kg gradual in 2 divided doses increases from $4,5 \mathrm{mg} / \mathrm{kg}$ from the first week to 7.5 and $10 \mathrm{mg} / \mathrm{kg}$ to the last week. In addition to Aerosolized 13 cis retinoic acid in gradual 2 divided doses increases from $0.2 \mathrm{mg} / \mathrm{kg} / \mathrm{day}$ to $4 \mathrm{mg} / \mathrm{kg} / \mathrm{day}$ as inhaled

Page 5/10 
retinoic acid therapy for 30 days. Moreover, the patients will receive Echinacea Purpurea Extract Capsules Polyphenols Chicoric Acid. Echinacea phytochemical profile Each tablet is comprised of the equivalent of $1275 \mathrm{mg}$ of echinacea root, as follows: A) Echinacea purpurea - $675 \mathrm{mg}$ root yields $112.5 \mathrm{mg}$ dried extract, standardized to contain $2.1 \mathrm{mg}$ alkamides. Each batch of tablets contain choric acid $=3.4$ to $8.5 \mathrm{mg} /$ tablet. Furthermore, the patients will receive Cholecalciferol(Vitamin D) Intramuscular injection of 600,000 units of Cholecalciferol for 2 doses given at week 0 and week 4

Active Comparator: All trans retinoic acid, Minocycline, Chicroic Acid and Vitamin D For (COVID-19 and MDR- TB)

50 subjects with confection of COVID-19 and multidrug-resistant tuberculosis (MDR-TB) will be randomly assigned to receive A) Minocycline i.v. 4,5 mg/kg gradual in 2 divided doses increases from $4,5 \mathrm{mg} / \mathrm{kg}$ from the first week to $7.5 \mathrm{and} 10 \mathrm{mg} / \mathrm{kg}$ to the last week. In addition to Aerosolized All trans retinoic acid in gradual 2 divided doses increases from $0.2 \mathrm{mg} / \mathrm{kg} / \mathrm{day}$ to $4 \mathrm{mg} / \mathrm{kg} / \mathrm{day}$ as inhaled retinoic acid therapy for 30 days. Moreover, the patients will receive Echinacea Purpurea Extract Capsules Polyphenols Chicoric Acid. Echinacea phytochemical profile Each tablet is comprised of the equivalent of $1275 \mathrm{mg}$ of echinacea root, as follows: A) Echinacea purpurea - $675 \mathrm{mg}$ root yields $112.5 \mathrm{mg}$ dried extract, standardized to contain $2.1 \mathrm{mg}$ alkamides. Each batch of tablets contain choric acid $=3.4$ to $8.5 \mathrm{mg} /$ tablet. Furthermore, the patients will receive Cholecalciferol(Vitamin D) Intramuscular injection of 600,000 units of Cholecalciferol for 2 doses given at week 0 and week 4

\section{Placebo Comparator: The standard therapy}

The standard therapy 100 infected patients with confection of COVID-19 and MDR-TB will receive the standard therapy for tuberculosis for 30 days

\section{Anticipated Results And Measurements Outcomes}

Primary Outcome Measure:

1. The primary outcome for this intent-to-treat trial is the time to Mycobacterium tuberculosis sputum culture conversion to negative and COVID-19 PCR result obtained with negative [Time Frame: Up to 4 weeks]

Secondary Outcome Measures:

1. Sputum culture result (positive or negative)\& COVID-19 PCR (positive or negative) [Time Frame: 2 weeks after therapy start date]

2. Immune function [Time Frame: one month]

Immune responses will be assessed by the superoxide bursts and proteolytic capacities of macrophages and monocytes. Activity indexes will be assessed by comparing mean substrate and calibration fluorescence.

3. Incidence of probable/confirmed TB; change in weight (mean weight gain) [Time Frame: one month up to 12 months]

4. Serum levels of CRP and ESR [Time Frame: one month]

5. All-cause mortality rate [Time Frame: one month]

6. Qualitative (positive/negative) and quantitative (mean change in counts) reversion of the T-cell assay [Time Frame: one month]

7. Absolute lymphocyte counts (CD4,CD8 and CD25+FOXP3+ Regulatory T) [Time Frame: one month]

8. Measurement of cytokine (IFN-gamma and alpha, IL-6, IL-10, TNF-alpha, TGF-beta) levels produced in response to M. tb. [Time Frame: one month]

9. Vitamin D status [Time Frame: one month]

Serum 25(OH)D concentrations

10. Retinoic acid status [Time Frame: one month]

Serum Retinoic acid concentrations

11. Minocycline status [Time Frame: one month]

Serum Minocycline concentrations

12. Serum indoleamine 2,3-dioxygenase (IDO) enzyme status [Time Frame: one month]

Page 6/10 
Serum indoleamine 2,3-dioxygenase (IDO) enzyme concentration

13. Chicoric Acid status [Time Frame: one month]

Serum Chicoric Acid concentrations

ETHICAL CONCIDERATION • Before conducting present study, the approval of the ethics committee and the research council of

Both Kafr-Elshiekh university ,Faculty of Medicine and National committee of Bioethics (H-01-R-009), Riyadh, Saudia Arabia. Written consent will be taken from the study participants (Patients \& control) after explaining the aim of the study to them.

- All research steps will be recorded in IRCT. - Written consent is obtained from all patients before entering the study. $•$ We will provide patients with complete and clear information about the research process. At each phase of the study, patients are allowed to leave the study. - The medical record of all patients are fully preserved

\section{Declarations}

\section{Conflict of Interest Statement}

The author declares that the research was conducted in the absence of any commercial or financial relationships that could be construed as a potential conflict of interest

\section{References}

1-Lim SS, Vos T, Flaxman AD, et al . A comparative risk assessment of burden of disease and injury attributable to 67 risk factors and risk factor clusters in 21 regions, 1990-2010: a systematic analysis for the Global Burden of Disease Study 2010. Lancet 2012; 380: 22242260

2-Stosic, M., Vukovic, D., Babic, D. et al. Risk factors for multidrug-resistant tuberculosis among tuberculosis patients in Serbia: a casecontrol study. BMC Public Health 18, 1114 (2018). https://doi.org/10.1186/s12889-018-6021-5.

3-World Health Organization. Global tuberculosis report 2013. Geneva, Switzerland, 2013

4-East African/British Medical Research Council. Results at 5 years of a controlled comparison of a 6-month and a standard 18-month regimen of chemotherapy for pulmonary tuberculosis. Am Rev Respir Dis 1977; 116: 3-8.

5-Stop TB Partnership Civil society-led TB/COVID-19 Working Group. The impact of COVID-19 on the TB epidemic: a community perspective. Geneva, Switzerland: Stop TB Partnership, 2020.

http://www.stoptb.org/assets/documents/resources/publications/acsm/Civil\%20Society\%20Report\%20on\%20TB\%20and\%20COVID.pdf? fbclid=IwAR3SOY4kyBs5a_35HleUhcvwRIWspePA4vVHESqcQxio7G4irivJ90cSU8k Accessed November 2020

6-WHO. Global Tuberculosis Report 2019.

7-A profile of a retrospective cohort of 22 patients with COVID-19 and active/treated tuberculosis. Gupta N, Ish P, Gupta A, Malhotra N, Caminero JA, Singla R, Kumar R, Yadav SR, Dev N, Agrawal S, Kohli S, Sen MK, Chakrabarti S, Gupta NK Eur Respir J. 2020 Nov; 56(5):

8-Active tuberculosis, sequelae and COVID-19 co-infection: first cohort of 49 cases.Tadolini M, Codecasa LR, García-García JM, Blanc FX, Borisov S, Alffenaar JW, Andréjak C, Bachez P, Bart PA, Belilovski E, Cardoso-Landivar J, Centis R, D'Ambrosio L, Luiza De Souza-Galvão M, Dominguez-Castellano A, Dourmane S, Fréchet Jachym M, Froissart A, Giacomet V, Goletti D, Grard S, Gualano G, Izadifar A, Le Du D, Marín Royo M, Mazza-Stalder J, Motta I, Ong CWM, Palmieri F, Rivière F, Rodrigo T, Silva DR, Sánchez-Montalvá A, Saporiti M, Scarpellini P, Schlemmer F, Spanevello A, Sumarokova E, Tabernero E, Tambyah PA, Tiberi S, Torre A, Visca D, Zabaleta Murguiondo M, Sotgiu G, Migliori GBEur Respir J. 2020 Jul; 56(1):.

9-Risk Factors for Coronavirus Disease 2019 (COVID-19) Death in a Population Cohort Study from the Western Cape Province, South Africa. Western Cape Department of Health in collaboration with the National Institute for Communicable Diseases, South Africa. Clin Infect Dis. 2021 Oct 5; 73(7):e2005-e2015. 
10-Demkina AE, et al. medRxiv. 2020. Risk factors for outcomes of COVID-19 patients: an observational study of 795572 patients in Russia. 2020.11.02.20224253.

11-McQuaid, C F et al. "The impact of COVID-19 on TB: a review of the data." The international journal of tuberculosis and lung disease : the official journal of the International Union against Tuberculosis and Lung Disease vol. 25,6 (2021): 436-446. doi:10.5588/ijtld.21.0148

12-Deshpande D, Pasipanodya JG, Srivastava S, Martin KR, Athale S, van Zyl J, Antiabong J, Koeuth T, Lee PS, Dheda K, Gumbo T. Minocycline Immunomodulates via Sonic Hedgehog Signaling and Apoptosis and Has Direct Potency Against Drug-Resistant Tuberculosis. J Infect Dis. 2019 Feb 23;219(6):975-985. doi: 10.1093/infdis/jiy587. PMID: 30597040.

13-Gautam, U. S., Foreman, T. W., Bucsan, A. N., Veatch, A. V., Alvarez, X., Adekambi, T., Golden, N. A., Gentry, K. M., Doyle-Meyers, L. A., Russell-Lodrigue, K. E., Didier, P. J., Blanchard, J. L., Kousoulas, K. G., Lackner, A. A., Kalman, D., Rengarajan, J., Khader, S. A., Kaushal, D., \& Mehra, S. (2018). In vivo inhibition of tryptophan catabolism reorganizes the tuberculoma and augments immune-mediated control of Mycobacterium tuberculosis. Proceedings of the National Academy of Sciences of the United States of America, 115(1), E62-E71. https://doi.org/10.1073/pnas.1711373114

14-Clemens, V., Regen, F., Le Bret, N. et al. Anti-inflammatory effects of minocycline are mediated by retinoid signaling. BMC Neurosci 19, 58 (2018). https://doi.org/10.1186/s12868-018-0460-x

15-Dutta RK, Kathania M, Raje M, Majumdar S. IL-6 inhibits IFN-y induced autophagy in Mycobacterium tuberculosis H37Rv infected macrophages. Int J Biochem Cell Biol. 2012 Jun;44(6):942-54. doi: 10.1016/j.biocel.2012.02.021. Epub 2012 Mar 7. PMID: 22426116.

16-Review Effects of vitamin a supplementation on immune responses and correlation with clinical outcomes. Villamor E, Fawzi WW Clin Microbiol Rev. 2005 Jul; 18(3):446-64.

17-Retinoic acids exert direct effects on T cells to suppress Th1 development and enhance Th2 development via retinoic acid receptors. Iwata M, Eshima Y, Kagechika H Int Immunol. 2003 Aug; 15(8):1017-25.

18-Retinoic acid and polyriboinosinic:polyribocytidylic acid stimulate robust anti-tetanus antibody production while differentially regulating type 1/type 2 cytokines and lymphocyte populations. Ma Y, Chen Q, Ross AC J Immunol. 2005 Jun 15; 174(12):7961-9.

19-Yamada, H., Mizuno, S., Ross, A. C., \& Sugawara, I. (2007). Retinoic acid therapy attenuates the severity of tuberculosis while altering lymphocyte and macrophage numbers and cytokine expression in rats infected with Mycobacterium tuberculosis. The Journal of nutrition, 137(12), 2696-2700. https://doi.org/10.1093/jn/137.12.2696

20-Abd-Nikfarjam B, Nassiri-Asl M, Hajiaghayi M, Naserpour Farivar T. Role of Chicoric Acid and 13-Cis Retinoic Acid in Mycobacterium tuberculosis Infection Control by Human U937 Macrophage. Arch Immunol Ther Exp (Warsz). 2018 Oct;66(5):399-406. doi: 10.1007/s00005-018-0511-0. Epub 2018 Apr 27. PMID: 29704020.

21-Talat, N., Perry, S., Parsonnet, J., Dawood, G., \& Hussain, R. (2010). Vitamin d deficiency and tuberculosis progression. Emerging infectious diseases, 16(5), 853-855. https://doi.org/10.3201/eid1605.091693

22-David A. Jolliffe, Davaasambuu Ganmaa, Christian Wejse, Rubhana Raqib, Md. Ahsanul Haq, Nawal Salahuddin, Peter K. Daley, Anna P. Ralph, Thomas R. Ziegler, Adrian R. Martineau. Adjunctive vitamin D in tuberculosis treatment: meta-analysis of individual participant data. European Respiratory Journal, 2019; 1802003 DOI: 10.1183/13993003.02003-2018

23-Esteban L, Eisman J, Gardiner E. Vitamin D receptor promoter and regulation of receptor expression. In: Feldman D, Pike JW, Glorieux FH, editors. Vitamin D. 2. New York: Elsevier/Academic Press; 2005. pp. 193-217

24-Cheng TY, Goodman GE, Thornquist MD, Barnett MJ, Beresford SA, LaCroix AZ, Zheng Y, Neuhouser ML. Estimated intake of vitamin D and its interaction with vitamin A on lung cancer risk among smokers. Int J Cancer. 2014 Nov 1;135(9):2135-45. doi: 10.1002/ijc.28846. Epub 2014 Mar 24. PMID: 24622914; PMCID: PMC4293152.

25. World Health Organization. Global Tuberculosis Reports. (2020). Available online at: https://www.who.int/teams/global-tuberculosisprogramme/tb-reports (accessed December 19, 2020).

26-Tadolini M, Codecasa L, García-García J, Blanc F, Borisov S, Alffenaar J, et al. Active tuberculosis, sequelae and COVID-19 co-infection: first cohort of 49 cases. Eur Respir J. (2020) 56:2002328. doi: 10.1183/13993003.02328-2020

Page $8 / 10$ 
27. Promislow DEL. A geroscience perspective on COVID-19 mortality. Js Gerontol Ser A Biol Sci Med Sci. (2020) 75:e30-3. doi: 10.1093/gerona/glaa094 PubMed Abstract | CrossRef Full Text | Google Scholar

28. Zhou F, Yu T, Du R, Fan G, Liu Y, Liu Z, et al. Clinical course and risk factors for mortality of adult inpatients with COVID-19 in Wuhan, China: a retrospective cohort study. Lancet. (2020) 395:1054-62. doi: 10.1016/S0140-6736(20)30566-3

29-Guan WJ, Liang WH, Zhao Y, Liang HR, Chen ZS, Li YM, et al. Comorbidity and its impact on 1590 patients with COVID-19 in China: a nationwide analysis. Eur Respir J. (2020) 55:2001227. doi: 10.1183/13993003.01227-2020

30-World Health Organization. WHO Coronavirus Disease (COVID-19) Dashboard. Available online at: https://covid19.who.int/table (accessed December 19, 2020).

31. Callender L, Curran M, Bates S, Mairesse M, Weigandt J, Betts $C$. The impact of pre-existing comorbidities and therapeutic interventions on COVID-19. Front Immunol. (2020) 11:1991. doi: 10.3389/fimmu.2020.01991

32-Goodman \& Gilman's. The pharmacological basis of therapeutics, 13e | Access medicine. New York City: McGraw-Hill Medical. 2018

33-Garrido-Mesa N, Zarzuelo A, Gálvez J. Minocycline: far beyond an antibiotic. Br J Pharmacol. 2013;169:337-352.

34-Garrido-Mesa N, Zarzuelo A, Gálvez J. Minocycline: far beyond an antibiotic. Br J Pharmacol. 2013;169:337-352.

35-Szeto GL, Brice AK, Yang H-C, et al. Minocycline attenuates HIV infection and reactivation by suppressing cellular activation in human CD4+ T cells. J Infect Dis. 2010;201:1132-1140.

36-Seabrook TJ, Jiang L, Maier M, et al. Minocycline affects microglia activation, abeta deposition, and behavior in APP-tg mice. Glia. 2006;53:776-782.

37-Enose-Akahata Y, Matsuura E, Tanaka Y, et al. Minocycline modulates antigen-specific CTL activity through inactivation of mononuclear phagocytes in patients with HTLV-I associated neurologic disease. Retrovirology. 2012;9:16.

38-Steinberg J, Halter J, Schiller $\mathrm{H}$, et al. Chemically modified tetracycline prevents the development of septic shock and acute respiratory distress syndrome in a clinically applicable porcine model. Shock Augusta Ga. 2005;24:348-356.

39-Sadowsky D, Nieman G, Barclay D, et al. Impact of chemically-modified tetracycline 3 on intertwined physiological, biochemical, and inflammatory networks in porcine sepsis/ARDS. Int J Burns Trauma. 2015;5:22-35

40-Zhou Y-Q, Liu D-Q, Chen S-P, et al. Minocycline as a promising therapeutic strategy for chronic pain. Pharmacol Res. 2018;134:305310.

41-Rosenblat JD, Mclntyre RS. Efficacy and tolerability of minocycline for depression: A systematic review and meta-analysis of clinical trials. J Affect Disord. 2018;227:219-225.

42-Wu C., Chen X. Risk factors associated with acute respiratory distress syndrome and death in patients with coronavirus disease 2019 pneumonia in Wuhan, China. JAMA Intern. Med. 2020;13

43-Dey, D., Borkotoky, S., \& Banerjee, M. (2020). In silico identification of Tretinoin as a SARS-CoV-2 envelope (E) protein ion channel inhibitor. Computers in biology and medicine, 127, 104063. https://doi.org/10.1016/j.compbiomed.2020.104063

44-Morita T, Miyakawa K, Jeremiah SS, Yamaoka Y, Sada M, Kuniyoshi T, Yang J, Kimura H, Ryo A. All-Trans Retinoic Acid Exhibits Antiviral Effect against SARS-CoV-2 by Inhibiting 3CLpro Activity. Viruses. 2021 Aug 23;13(8):1669. doi: 10.3390/v13081669. PMID: 34452533; PMCID: PMC8402917.

45-Riva, L., Yuan, S., Yin, X. et al. Discovery of SARS-CoV-2 antiviral drugs through large-scale compound repurposing. Nature 586, 113119 (2020). https://doi.org/10.1038/s41586-020-2577-1

46-Gundogdu M, Dere G. Is systemic isotretinoin use a risk factor for coronavirus disease 2019 (COVID-19)? J Cosmet Dermatol. 2021 Jun;20(6):1568-1570. doi: 10.1111/jocd.14044. Epub 2021 Mar 14. PMID: 33682290; PMCID: PMC8251030. 
47-Abdelmaksoud A, Patil A, Dursun R, Temiz SA, Ayhan E, Goldust M, Vestita M. Could isotretinoin be a protective agent against COVID19?: A dermatologist perspective. J Cosmet Dermatol. 2021 Aug;20(8):2394-2395. doi: 10.1111/jocd.14239. Epub 2021 Jun 6. PMID: 34008285; PMCID: PMC8242528.

48-Hamouda Elgarhy L. Could patients taking isotretinoin therapy be immune against SARS-CoV-2? Dermatol Ther. 2020 Jul;33(4):e13573. doi: 10.1111/dth.13573. Epub 2020 May 29. PMID: 32406143; PMCID: PMC7272898.

49-Demir M, Demir F, Aygun H. Vitamin D deficiency is associated with COVID-19 positivity and severity of the disease. J Med Virol. 2021 May;93(5):2992-2999. doi: 10.1002/jmv.26832. Epub 2021 Feb 9. PMID: 33512007; PMCID: PMC8013436.

50-Aranow C. Vitamin D and the immune system. J Investig Med. 2011;59(6):881-886.

51-Khoo AL, Chai L, Koenen H, Joosten I, Netea M, van der Ven A. Translating the role of vitamin D3 in infectious diseases. Crit Rev Microbiol. 2012;38(2):122-135

52-Karahan S, Katkat F. Impact of Serum $25(\mathrm{OH})$ Vitamin D level on mortality in patients with COVID-19 in Turkey. J Nutr Health Aging. 2020:1-8

53-Entrenas Castillo M, Entrenas Costa LM, Vaquero Barrios JM, et al. Effect of calcifediol treatment and best available therapy versus best available therapy on intensive care unit admission and mortality among patients hospitalized for COVID-19: A pilot randomized clinical study. J Steroid Biochem Mol Biol. 2020;203:105751.

54-J. L, B. J, C.E. W, G. R, T.M. C, Antiviral activity of metal chelates of caffeic acid and similar compounds towards herpes simplex, VSVEbola pseudotyped and vaccinia viruses, Antivir. Res. 160 (2018) 143-150.

55-S.U. L, C.G. S, C.K. L, Y.S. L, Caffeoylglycolic and caffeoylamino acid derivatives, halfmers of L-chicoric acid, as new HIV-1 integrase inhibitors, Eur. J. Med. Chem. 42 (10) (2007) 1309-1315.

56-L.Z. S, N.L. C, S.C. M, The American coneflower: a prophylactic role involving nonspecific immunity, J. Alternative Compl. Med. 5 (5) (1999) 437-446.

57-A. Wacker, W. Hilbig, Virushemmung mit Echinacea purpurea, Planta Med. 33 (1) (1978) 89-102. 